\title{
Gramíneas bioindicadoras da presença de flúor em regiões tropicais
}

\author{
MARCO ANTONIO OLIVA ${ }^{1,2}$ e JOSÉ GUILHERME DE FIGUEIREDO ${ }^{1}$
}

(recebido: 4 de março de 2004; aceito: 17 de fevereiro de 2005)

\begin{abstract}
Fluoride bioindicator grasses for tropical regions). Ten species of tropical grasses, Andropogon gayanus Kunth cv. Planaltina, Panicum maximum Jacq. cv. Colonião, Panicum maximum Jacq. cv. Tobiatã, Chloris gayana Kunth, Eragrostis curvula (Schrad.) Nees, Paspalum notatum Flug. cv. Pensacola, Hiparrhenia rufa (Nees) Stapf., Melinis minutiflora Pal. de Beauv., Brachiaria decumbens Stapf., Brachiaria humidicola (Rendle) Schw. and Lolium multiflorum ssp. italicum var. lema were treated with simulated rain containing 5,10 , and $15 \mathrm{~g} . \mathrm{m}^{-3}$ of fluoride, in order to evaluate their relative sensitivity and potential as bioindicator for fluoride under tropical conditions. The plants were cultivated according to standardized techniques for active bioindication. The development of visible damages, the accumulation and distribution of fluoride and the dry weight yield were used to assess the effects of the element on the plants. All studied species showed distinct pattern of fluoride accumulation, fluorine contents above the accepted toxic levels for sensitive species $\left(30 \mu \mathrm{g} . \mathrm{g}^{-1}\right)$ and a good correlation between fluoride content and the phytotoxicity index, except for Brachiaria humidicola. Chloris gayana and Panicum maximum cv. Colonião were the species that better adapted to the standardized cultivation techniques, based on their growth rates, sprout homogeneity and fast appearance of visible symptoms (chlorosis and necrosis). Fluoride effects on stomatal conductance, $\mathrm{CO}_{2}$ assimilation and dry weight yield were also evaluated in these two species, with potential for bioindication use. The results indicate Panicum maximum cv Colonião as a responsive bioindicator while Chloris gayana as a tolerant species, similarly to L. multiflorum.
\end{abstract}

Key words - accumulation, chlorosis, fluoride, photosynthesis, responsive bioindicator, tolerance

RESUMO - (Gramíneas bioindicadoras da presença de flúor em regiões tropicais). Dez espécies de gramíneas tropicais Andropogon gayanus Kunth cv. Planaltina, Panicum maximum Jacq. cv. Colonião, Panicum maximum Jacq. cv. Tobiatã, Chloris gayana Kunth, Eragrostis curvula (Schrad.) Nees, Paspalum notatum Flug. cv. Pensacola, Hiparrhenia rufa (Nees.) Stapf., Melinis minutiflora Pal. de Beauv., Brachiaria decumbens Stapf., Brachiaria humidicola (Rendle) Schw. e Lolium multiflorum ssp. italicum var. lema foram tratadas com chuva simulada, contendo 5, 10 e $15 \mathrm{~g} . \mathrm{m}^{-3}$ de flúor, visando avaliar a sensibilidade relativa e o potencial bioindicador de cada espécie para regiões tropicais. As plantas foram cultivadas sob técnicas padronizadas para a bioindicação ativa. Os efeitos do flúor foram avaliados pelos danos visuais, acúmulo e distribuição do flúor nas plantas e produção de matéria seca. Todas as espécies mostraram padrões de acúmulo diferenciados, teores acima do nível considerado tóxico para plantas sensíveis $\left(30 \mu \mathrm{g} . \mathrm{g}^{-1}\right)$ e boa relação entre o teor de flúor na planta e os índices de fitotoxicidade, com exceção de Brachiaria humidicola. As espécies que melhor se adaptaram às técnicas de cultivo padronizado, por suas taxas de crescimento, homogeneidade das rebrotas e velocidade de resposta (necrose e clorose) foram Chloris gayana e Panicum maximum cv. Colonião. Nestas duas espécies com maior potencial de bioindicação, foram avaliados também os efeitos do flúor sobre a condutância estomática, a assimilação de $\mathrm{CO}_{2}$ e a produção de matéria seca. Os resultados obtidos caracterizam o Panicum maximum cv. Colonião como bioindicador de resposta e Chloris gayana como espécie tolerante, semelhante a L. multiflorum.

Palavras-chave - acúmulo, bioindicador de resposta, clorose, fluoreto, fotossíntese, tolerância

\section{Introdução}

A degradação de diversas formações vegetais no entorno de alguns pólos industriais no Brasil encontra-se, significativamente, relacionada à presença de flúor na atmosfera ou no solo, em conseqüência da atividade de indústrias de fertilizantes fosfatados e de usinas de alumínio. Alguns dos efeitos sobre as espécies e a

\footnotetext{
1. Universidade Federal de Viçosa, Departamento de Biologia Vegetal, 36571-000 Viçosa, MG, Brasil.

2._Autor para correspondência: moliva@ufv.br
}

vegetação tropical foram descritos por Weinstein \& Hansen (1988), Alves \& Oliva (1993), Klumpp et al. (1994), Arndt et al. (1995), Klumpp et al. (1996 a, b, c), Oliva et al. (1997), Domingos et al. (1998), Silva et al. (2000) e Domingos et al. (2004). O monitoramento das emissões de flúor, quando existente, emprega métodos físico-químicos de alto custo e de difícil aplicação, ou biológicos que, em muitos casos, se reduzem a meras observações de injúrias em plantas ou animais. O uso de organismos indicadores, como abordagem alternativa e/ou complementar em programas de monitoramento de poluição ambiental, permite uma interpretação melhor das relações ecológicas envolvidas. Para isso, um pré- 
requisito essencial é que as espécies a serem empregadas como indicadores ativos ou passivos sejam de ampla distribuição regional e de sensibilidade diferenciada ao poluente (Arndt \& Schweizer 1991).

Em diversos países, tem sido empregada uma metodologia padronizada que utiliza Lolium multiflorum ssp. italicum, espécie de gramínea acumuladora de flúor (Ruthsatz \& Wey 1991) no monitoramento de áreas poluídas com esse ânion (VDI-Richtlinie 1982). Entretanto, Ellenberg (1991) questiona a universalidade do seu uso, uma vez que condições ambientais locais podem alterar as propriedades ecofisiológicas de L. multiflorum e mascarar a sintomatologia específica ao flúor, e sugere o emprego de espécies da flora local. Há, portanto, a necessidade de se conhecer a sensibilidade ao flúor de diversas espécies de gramíneas nativas ou adaptadas a regiões tropicais. Suas possibilidades de serem utilizadas como indicadores de reação, biosensores, biomarcadores, bioacumuladores ou biointegradores (Temmerman et al. 2004) dependerá da capacidade de adaptação às técnicas de cultivo padronizado, da especificidade das respostas fisiológicas, bioquímicas e moleculares ao poluente, bem como de seus mecanismos de resistência. Nesses termos, a ampla distribuição geográfica de diversas espécies de gramíneas nas regiões tropical e subtropical e as altas taxas de crescimento da maioria delas sugerem a possibilidade do seu emprego como indicadoras da presença de flúor.

Nesse contexto, procurou-se avaliar o potencial indicativo da presença de fluoreto em 10 espécies de gramíneas forrageiras de ampla distribuição geográfica na região tropical, de acordo com suas sensibilidades ao poluente, suas possibilidades de cultivo e facilidade de avaliação em uma bioindicação ativa.

\section{Material e métodos}

Plantas de gramíneas forrageiras tropicais, Andropogon gayanus Kunth cv. Planaltina (capim gambá), Panicum maximum Jacq. cv. Colonião, Panicum maximum Jacq. cv. Tobiatã, Chloris gayana Kunth (capim-rhodes), Eragrostis curvula (Schrad.) Nees (capim-chorão), Paspalum notatum Flug. cv. Pensacola, Hiparrhenia rufa (Nees.) Stapf. (capimjaragua), Melinis minutiflora Pal. de Beauv. (capim-gordura), Brachiaria decumbens Stapf. e Brachiaria humidicola (Rendle) Schw. foram cultivadas seguindo o protocolo descrito pela VDI-Richtlinie 3792 (1982) para Lolium multiflorum Lam. ssp. italicum var. lema (azevém), espécie empregada como referência no monitoramento de flúor. Modificações do protocolo foram feitas no substrato e na aplicação do flúor na forma de chuva simulada. As sementes de Lolium multiflorum Lam. ssp. italicum var. lema eram provenientes do Institut für Pflanzenökologie da JustusLiebig Universität, Giessen, Alemanha.

O substrato foi adaptado, isto é, corrigido e fertilizado para as condições de cultivo de gramíneas forrageiras tropicais. Em todos os ensaios, utilizou-se como padrão um Latossolo Vermelho-Amarelo (LV), coletado do horizonte C na região de Viçosa-MG, misturado com areia lavada em uma razão 3:1. Para correção do pH do solo foi utilizado calcário dolomítico, PRNT $=80 \%$ em quantidade equivalente a 3,8 t.ha $^{-1}$. Todas as espécies receberam adubação inicial com superfosfato simples, cloreto de potássio e esterco de gado, em quantidades equivalentes a $60 \mathrm{~kg}$. ha- ${ }^{-1} \mathrm{de}_{2} \mathrm{O}_{5}, 30 \mathrm{~kg} \mathrm{ha}^{-1}$ de $\mathrm{K}_{2} \mathrm{O}$ e 60 t.ha $^{-1}$, respectivamente (tabela 1). Após cada corte e o desbaste definitivo, cada espécie recebeu uma adubação de cobertura adicional com solução de Hoagland, na base de $50 \mathrm{ml}^{\text {.vaso }}{ }^{-1}$. Exceções foram as espécies $P$. notatum e $B$. humidicola que receberam duas adubações a mais, devido a seu prolongado tempo de cultivo.

Cada gramínea foi semeada em vasos plásticos contendo $0,0018 \mathrm{~m}^{3}$ de substrato e mantida em casa de vegetação. Assim que as plântulas atingiram cerca de 20 a $30 \mathrm{~mm}$ de altura, foi realizado um desbaste para deixar um número definido de

Tabela 1. Características físicas e químicas do substrato (Latosolo vermelho) utilizado no cultivo das gramíneas após adubação.

Table 1. Physical and chemical characteristics of the Redyellow Latosol used to cultivate the grass species, after fertilization.

\section{Características químicas}

\begin{tabular}{lc}
\hline $\mathrm{pH}$ in $\mathrm{H}_{2} \mathrm{O}(1: 2,5)$ & 5,7 \\
$\mathrm{Al}^{3+}\left(\mathrm{cmol}_{\mathrm{c}} \cdot \mathrm{dm}^{-3}\right)$ & 0,0 \\
$\mathrm{H}+\mathrm{Al}\left(\mathrm{cmol}_{\mathrm{c}} \cdot \mathrm{dm}^{-3}\right)$ & 2,4 \\
$\mathrm{Ca}^{2+}\left(\mathrm{cmol}_{\mathrm{c}} \cdot \mathrm{dm}^{-3}\right)$ & 2,8 \\
$\mathrm{Mg}^{2+}\left(\mathrm{cmol}_{\mathrm{c}} \cdot \mathrm{dm}^{-3}\right)$ & 0,3 \\
$\mathrm{P}\left(\mathrm{mg}_{\mathrm{dm}}^{-3}\right)$ & 0,3 \\
$\mathrm{~K}\left(\mathrm{mg} \cdot \mathrm{dm}^{-3}\right)$ & 16,9 \\
$\mathrm{SB}$ & 238 \\
$\mathrm{CEC}-\mathrm{pH} 7$ & 3,74 \\
$\mathrm{~V}(\%)$ & 60,9 \\
Organic carbon $\left(\mathrm{g}^{-3} \mathrm{hg}^{-1}\right)$ & - \\
\hline
\end{tabular}

Características físicas

$\begin{array}{lc}\text { Coarse sand }\left(\mathrm{g} \cdot \mathrm{hg}^{-1}\right) & 50 \\ \text { Fine sand }\left(\mathrm{g} \cdot \mathrm{hg}^{-1}\right) & 3 \\ \text { Silt }\left(\mathrm{g} \cdot \mathrm{hg}^{-1}\right) & 10 \\ \text { Clay }\left(\mathrm{g} \cdot \mathrm{hg}^{-1}\right) & 37 \\ \text { Soil texture } & \text { Sandy - clay } \\ \text { Particle density }\left(\mathrm{g}^{-\mathrm{cm}^{-3}}\right) & 2,58 \\ \text { Bulk density }\left(\mathrm{g} \cdot \mathrm{hg}^{-1}\right) & 1,15\end{array}$


plantas de cada espécie por vaso. Com a finalidade de padronizar os cultivos em cada unidade experimental e antes que as plantas fossem expostas ao fluoreto, todas as espécies sofreram um corte aproximadamente aos 27 e outro aos 43 dias após a emergência quando atingiam, em média, $250 \mathrm{~mm}$ de altura. O tempo total de cultivo, da semeadura ao início dos tratamentos, variou entre as espécies de seis a oito semanas, tempo suficiente para se obter rebrotas vigorosas. As adaptações das espécies às técnicas de cultivo a ser aplicado em bioindicação ativa foi avaliada pela determinação do crescimento inicial Wi, o crescimento das rebrotas Wreb e o crescimento total. O crescimento inicial, Wi corresponde à produção da matéria seca colhida de cada espécie no momento do primeiro corte, o crescimento das rebrotas Wreb corresponde ao material vegetal colhido acima do nível do corte em cada rebrota e o crescimento total corresponde à soma dos crescimentos $\left(\mathrm{Wt}=\mathrm{Wi}+\mathrm{Wreb}_{1}+\mathrm{Wreb}_{2}+\ldots \ldots \mathrm{Wreb}_{\mathrm{n}}\right.$.

As plantas de todas as espécies foram expostas a quatro doses de flúor $\left(0,5,10\right.$ e $\left.15 \mathrm{~g} . \mathrm{m}^{-3}\right)$ aplicadas na forma de chuva simulada de fluoreto de potássio, em um sistema adaptado por Alves et al. (1990). As simulações de chuva foram realizadas diariamente, com duração de 15 minutos, por um período de 20 dias. A freqüência e a intensidade dessas simulações resultaram em uma precipitação total de $300 \mathrm{~mm}$. Antes e depois da exposição, as plantas ficaram expostas durante 15 minutos a uma irradiância de $14,7 \mu \mathrm{mol}$ de fótons. $\mathrm{m}^{-2} \cdot \mathrm{s}^{-1}$. e temperatura média de $26 \pm 2^{\circ} \mathrm{C}$.

No fim de cada tratamento foi realizada uma avaliação visual do grau de injúria causado pelo $\mathrm{F}^{-}$e determinado o índice de fitotoxicidade a partir de uma média de três plantas de cada espécie, de acordo com a seguinte escala de pontuação: $0=$ planta sem injúria, $1=$ início de clorose, $2=$ clorose generalizada, $3=$ início de necrose e $4=$ clorose e necrose mais acentuada (Bustamante et al. 1993).

$\mathrm{O}$ material vegetal foi dividido em parte aérea (PA) (rebrota e soca) e sistema radicular (SR) sendo considerado rebrota o material vegetal colhido acima da altura de corte (50 $\mathrm{mm}$ sobre o solo) e soca, o caule (rizoma) mais a parte aérea remanescente após o corte. Amostras de cada uma das quatro repetições foram lavadas com água deionizada, secas a $70^{\circ} \mathrm{C}$, reduzidas, em moinho do tipo Wiley, a partículas com dimensões inferiores a $1 \mathrm{~mm}$ e alíquotas de $0,5 \mathrm{~g}$ foram submetidas a lixiviação durante 16 horas em ácido perclórico $0,1 \mathrm{M}$. O teor de fluoreto foi determinado no extrato resultante da lixiviação utilizando-se um eletrodo íon-específico (Orion 94-09), após ajuste da força iônica da solução e descomplexação do fluoreto (García-Ciudad et al. 1985).

Após as avaliações de crescimento, da injúria e do acúmulo de $\mathrm{F}^{-}$no material vegetal, foram escolhidas as duas espécies que melhor se adaptaram tanto às condições de cultivo para bioindicação, quanto ao grau de sensibilidade relativa ao $\mathrm{F}^{-}$. Juntamente com $L$. multiflorum, mantida como espécie de referência, foram selecionadas $P$. maximum cv. Colonião e $C$. gayana. As trocas gasosas foram avaliadas nas três espécies no segmento mediano de folhas jovens totalmente desenvolvidas mediante o emprego de um analisador de gases por infravermelho (LCA-4, Analytical Development Co, Hoddesdon, UK), entre 8:00 e 10:00 h, em sala climatizada $\left(22^{\circ} \mathrm{C}\right)$ sob irradiância de $1000 \mu \mathrm{mol} \cdot \mathrm{m}^{-2} \cdot \mathrm{s}^{-1}$. A partir dos resultados, foram calculadas as taxas de fotossíntese líquida (A) e a condutância estomática ao vapor de água $\left(\mathrm{g}_{\mathrm{s}}\right)$.

O delineamento experimental para todos os ensaios foi inteiramente casualizado com quatro repetições sendo que as médias foram submetidas ao teste de Tukey, a 5\% de probabilidade, para comparação.

\section{Resultados}

Os valores de Wi, Wreb e Wt mostraram a capacidade de adaptação de cada espécie às técnicas de cultivo adotadas. As espécies $P$. maximum cv. Colonião, $P$. maximum cv. Tobiatã e $C$. gayana mostraram-se promissoras para bioindicação, em função dos altos valores de Wi e Wt (tabela 2). Já as plantas de A. gayanus e B. decumbens apresentaram germinação lenta e heterogênea, crescimento em altura e produção de matéria seca irregular $(\mathrm{CV}=37 \%$ e $14 \%$, respectivamente) e valores de Wi e Wt menores. Plantas de $H$. rufa também apresentaram crescimento inicial lento, porém, com rebrotas rápidas, resultando em plantas uniformes e vigorosas. As espécies de $P$. notatum e $B$. humidicola que apresentaram a menor e maior produção de matéria seca inicial, 0,21 e 2,48 g.vaso $^{-1}$, respectivamente, não se adaptaram ao manejo devido ao lento crescimento da rebrota, permitindo apenas um corte durante todo o período de cultivo. Finalmente, L. multiflorum mostrou um alto valor de Wi e Wreb no início, porém, após o segundo corte, apresentou significativo decréscimo na sua capacidade de rebrota (tabela 2).

Uma vez expostas ao $\mathrm{F}^{-}$, as gramíneas mostraram ampla variação na capacidade de acumular o poluente (figura 1). Em todas as espécies, os incrementos de $\mathrm{F}^{-}$ foram significativamente maiores na parte aérea das plantas, especialmente na rebrota, com exceção de B. humidicola e H. rufa, que acumularam mais $\mathrm{F}^{-}$na soca (figura 2). Brachiaria humidicola e E. curvula foram as espécies com menor $\left(19,8 \mu \mathrm{g} \cdot \mathrm{g}^{-1} \mathrm{MS}\right)$ e maior (201,1 $\left.\mu \mathrm{g} \cdot \mathrm{g}^{-1} \mathrm{MS}\right)$ conteúdo de $\mathrm{F}^{-}$, respectivamente. Lolium multiflorum, considerada uma espécie resistente e acumuladora, foi tomada como referência e, a partir dela, foi avaliada a capacidade de acumular flúor das outras gramíneas quando submetidas à dose de $15 \mathrm{~g} \cdot \mathrm{m}^{-3}$. Em geral, as espécies testadas se localizaram numa faixa intermediária junto com $L$. multiflorum e com uma certa tolerância à presença do flúor nos seus tecidos. Das 
Tabela 2. Comparação dos valores médios dos dias após a emergência (DAE) até o primeiro corte, produção de matéria seca inicial (Wi), produção da primeira rebrota (Wreb), altura das plantas no momento do segundo corte e produção total das gramíneas (Wt), cultivadas em Latossolo Vermelho-Amarelo (LV)

Table 2. Comparision of means of days after the emergence (DAE) until the first cutting, dry matter yield (Wi), yield of the first sprout (Wsp), height of the plants at the second cutting and total yield (Wt) of all species cultivated in Red-Yellow Latosol.

\begin{tabular}{|c|c|c|c|c|c|c|}
\hline Espécies & $\begin{array}{l}\text { DAE até o } \\
\text { primeiro corte }\end{array}$ & $\begin{array}{c}\text { Wi } \\
\left(\text { g.vaso }{ }^{-1}\right)\end{array}$ & $\begin{array}{c}\text { Wreb } \\
\left(\text { g.vaso }{ }^{-1}\right)\end{array}$ & $\begin{array}{l}\text { Altura média no } \\
\text { secundo corte } \\
(\mathrm{mm})\end{array}$ & $\begin{array}{c}\mathrm{Wt} \\
\left(\text { g.vaso }{ }^{-1}\right)\end{array}$ & $\begin{array}{l}\mathrm{CV} \\
(\%)\end{array}$ \\
\hline A.gayanus & 21 & $0,76 \pm 0,09$ & $0,69 \pm 0,04$ & 160 & $1,47 \pm 0,14$ & 37 \\
\hline B. decumbens & 26 & $0,42 \pm 0,03$ & $1,11 \pm 0,05$ & 130 & $1,53 \pm 0,05$ & 14 \\
\hline B. humidicola & 78 & $2,48 \pm 0,11$ & - & - & $2,48 \pm 0,11$ & 18 \\
\hline C.gayana & 29 & $1,18 \pm 0,06$ & $1,08 \pm 0,07$ & 180 & $2,26 \pm 0,11$ & 18 \\
\hline E. curvula & 25 & $0,82 \pm 0,03$ & $1,33 \pm 0,04$ & 180 & $2,15 \pm 0,05$ & 9 \\
\hline H. rufa & 38 & $0,69 \pm 0,02$ & $0,94 \pm 0,03$ & 180 & $1,63 \pm 0,04$ & 10 \\
\hline L. multiflorum & 19 & $0,77 \pm 0,03$ & $0,48 \pm 0,01$ & 100 & $1,45 \pm 0,10$ & 28 \\
\hline M. minutiflora & 32 & $1,16 \pm 0,04$ & $1,42 \pm 0,04$ & 170 & $2,58 \pm 0,07$ & 11 \\
\hline P. maximum cv. Colonião & 28 & $1,58 \pm 0,02$ & $1,12 \pm 0,07$ & 150 & $2,70 \pm 0,08$ & 11 \\
\hline P. maximum cv. Tobiatã & 27 & $0,78 \pm 0,03$ & $1,36 \pm 0,04$ & 200 & $2,14 \pm 0,05$ & 9 \\
\hline P. notatum & 51 & $0,21 \pm 0,02$ & - & - & $0,21 \pm 0,02$ & 29 \\
\hline
\end{tabular}

espécies tropicais estudadas, as variedades de $P$. maximum cv. Colonião e P. maximum cv. Tobiatã acumularam altos teores de flúor, concomitantemente a um rápido desenvolvimento da sintomatologia, o que as caracteriza como espécies bioindicadoras de reação. $\mathrm{O}$ acúmulo do flúor, entretanto, mostrou características específicas. Enquanto a maioria das espécies acumulava flúor na rebrota, $B$. humidicola acumulava preferencialmente na soca e $L$. multiflorum, $C$. gayana e $H$. rufa mostraram um certo equilíbrio entre soca e rebrota (figura 2).

Na maioria das espécies, foi possível relacionar o grau de injúria, determinado mediante o índice de fitotoxicidade, com o acúmulo de $\mathrm{F}^{-}$na rebrota (figura 3). Pela extensão dessas injúrias, destacam-se, $P$. maximum cv. Colonião e $P$. notatum que, junto com M. minutiflora, foram as primeiras a mostrarem os efeitos do $\mathrm{F}^{-}$já no segundo dia após iniciados os tratamentos nas concentrações de 10 e 15 g.m $\mathrm{m}^{-3}$. Plantas das espécies $P$. maximum cv. Tobiatã, $B$. decumbens e E. curvula mostraram sintomatologia apenas no terceiro, quarto e oitavo dias, respectivamente, e L. multiflorum, $C$. gayana, A. gayanus e $H$. rufa não manifestaram qualquer dano visível. As injúrias foliares nas espécies afetadas foram semelhantes, iniciando-se como manchas cloróticas de forma e tamanhos irregulares que se concentraram nas margens e que evoluíram, rapidamente, para necrose apical. De uma clorose de cor verde opaco elas evoluíram para necrose marrom-escura. As gramíneas mais sensíveis como $P$. maximum cv. Colonião, $P$. notatum e $P$. maximum cv. Tobiatã apresentaram, no início das injúrias, manchas cloróticas de cor amarelo-opaca na nervura central, que necrosaram lentamente. Nas concentrações mais altas de $\mathrm{F}^{-}$, a injúria severa afetou a folha inteira e clorose e necroses tornaram-se generalizadas. Folhas recentemente expandidas como a segunda e terceira folhas a partir do ápice foram as mais sensíveis e as primeiras a mostrarem sintomas.

Os resultados mostrados anteriormente apontam duas espécies que poderiam ser empregadas em bioindicação ativa: P. maximum cv. Colonião e C. gayana. A primeira destacou-se pelo rápido e significativo crescimento da rebrota, alto acúmulo de $\mathrm{F}^{-}$ e sensibilidade ao poluente com inequívoca formação de injúria. A segunda poderia ser caracterizada como resistente, apresentando menor acúmulo de $\mathrm{F}^{-} \mathrm{e}$ ausência de injúrias aparentes. Mantendo-se L. multiflorum ssp. italicum como espécie de referência, foram avaliados em conjunto os efeitos de concentrações crescentes de $\mathrm{F}^{-}$sobre a condutância estomática $\left(\mathrm{g}_{\mathrm{s}}\right)$, a fotossíntese (A) e a produção de matéria seca. O efeito de diversas concentrações de $\mathrm{F}^{-}$na chuva simulada causou um decréscimo significativo $(\mathrm{p}<1 \%)$ de $\mathrm{g}_{\mathrm{s}}$, apenas em $P$. maximum cv. Colonião. Ao contrário do observado para essa espécie, em L. multiflorum e C. gayana houve uma leve tendência de aumento dos valores de $\mathrm{g}_{\mathrm{s}}$, o que pode indicar uma leve perda do controle da 


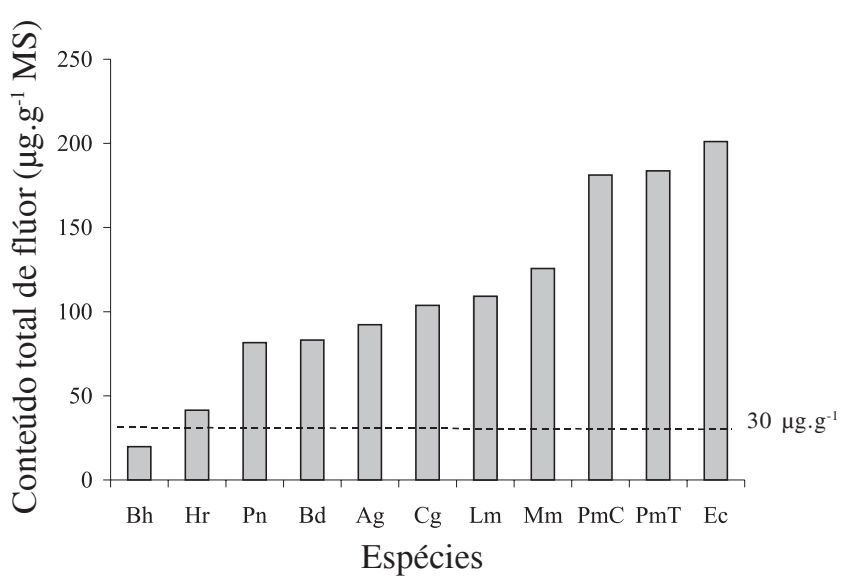

Figura 1. Conteúdo total de $\mathrm{F}^{-}$na matéria seca das rebrotas em espécies de gramíneas submetidas a chuvas simuladas com uma solução de $15 \mathrm{gF}^{-} \cdot \mathrm{m}^{-3}$ durante 20 dias. $\mathrm{Bh}=B$. humidicola, $\mathrm{Hr}=H$. rufa, $\mathrm{Pn}=P$. notatum, $\mathrm{Bd}=B$. decumbens, $\mathrm{Ag}=A$. gayanus, $\mathrm{Cg}=C$. gayana, $\mathrm{Lm}=$ L. multiflorum, $\mathrm{Mm}=$ M. minutiflora, $\mathrm{PmC}=P$. maximum cv. Colonião, $\mathrm{PmT}=P$. maximum $\mathrm{cv}$. Tobiatã, Ec $=$ E. curvula

Figure 1. $\mathrm{F}^{-}$total content on sprout dry matter of the grass species submitted to simulated rain, during 20 days, containing fluoride at $15 \mathrm{~g} \mathrm{~F}^{-} . \mathrm{m}^{-3} . \mathrm{Bh}=B$. humidicola, $\mathrm{Hr}=H$. rufa, $\mathrm{Pn}=P$. notatum, $\mathrm{Bd}=B$. decumbens, $\mathrm{Ag}=A$. gayanus, $\mathrm{Cg}=C$. gayana, $\mathrm{Lm}=L$. multiflorum, $\mathrm{Mm}=M$. minutiflora, $\mathrm{PmC}=P$. maximum cv. Colonião, $\mathrm{PmT}=P$. maximum cv. Tobiatã, Ec $=$ E. curvula

planta sobre os mecanismos de fechamento estomático $\mathrm{e}$, conseqüentemente, sobre as trocas gasosas (figura 4). De fato, a diminuição de $\mathrm{g}_{\mathrm{s}}$ causou em $P$. maximum $\mathrm{cv}$. Colonião uma queda significativa $(\mathrm{p}<1 \%)$ dos valores de A e em $C$. gayana o aumento de $\mathrm{g}_{\mathrm{s}}$ induziu incremento das taxas fotossintéticas (figura 5). A diminuição de $\mathrm{g}_{\mathrm{s}}$ e de A em $P$. maximum cv. Colonião causou uma progressiva queda na produção de matéria seca. Em C. gayana foi encontrada uma relação significativa $(p<1 \%)$ entre $g_{s}$ e $A$, mas seus efeitos sobre o acúmulo de matéria seca mostraram tendências contraditórias. As plantas de L. multiflorum não acusaram qualquer efeito do $\mathrm{F}^{-}$sobre a assimilação de $\mathrm{CO}_{2}$ em geral (figura 6).

\section{Discussão}

A bioindicação ativa é realizada por plantas de espécies com sensibilidade conhecida que, introduzidas numa área impactada, apresentam inequívocas respostas a um determinado poluente ou a um conjunto deles. Tal

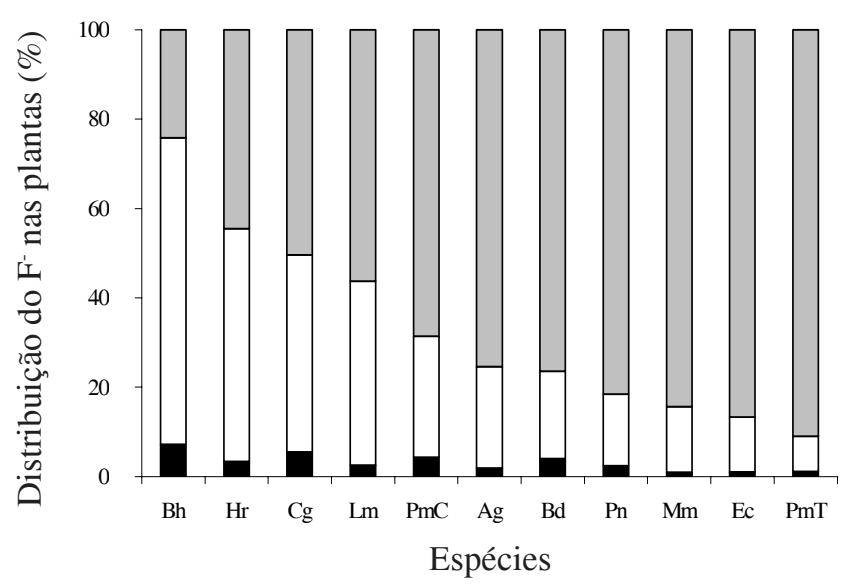

Figura 2. Distribuição relativa de $\mathrm{F}^{-}$na matéria seca das espécies de gramíneas submetidas a chuvas simuladas com uma solução de $15 \mathrm{~g} \mathrm{~F}^{-} \cdot \mathrm{m}^{-3}$ durante 20 dias. $\mathrm{Bh}=B$. humidicola, $\mathrm{Hr}=H$. rufa,$\quad \mathrm{Pn}=P$. notatum, $\mathrm{Bd}=B$. decumbens, $\mathrm{Ag}=$ A. gayanus, $\mathrm{Cg}=C$. gayana, $\mathrm{Lm}=L$. multiflorum, $\mathrm{Mm}=M$. minutiflora, $\mathrm{PmC}=P$. maximum cv. Colonião, $\mathrm{PmT}=$ P. maximum cv. Tobiatã, Ec $=$ E. curvula $. \mathbf{\square}=\mathrm{F}^{-}$raiz, $\square=\mathrm{F}^{-}$soca, $\square=\mathrm{F}^{-}$rebrota.

Figure 2. Relative distribution of $\mathrm{F}^{-}$on dry matter of the grass species submitted to simulated rain, during 20 days, containing fluoride at $15 \mathrm{~g} \mathrm{~F}^{-} \cdot \mathrm{m}^{-3} \mathrm{Bh}=B$. humidicola, $\mathrm{Hr}=H . \mathrm{rufa}$, $\mathrm{Pn}=P$. notatum, $\mathrm{Bd}=B$. decumbens, $\mathrm{Ag}=A$. gayanus, $\mathrm{Cg}=C$. gayana, $\mathrm{Lm}=L$. multiflorum, $\mathrm{Mm}=M$. minutiflora, $\mathrm{PmC}=P$. maximum cv. Colonião, $\mathrm{PmT}=P$. maximum cv. Tobiatã, Ec $=$ E. curvula . $\mathbf{\square}=\mathrm{F}^{-}$roots, $\square=\mathrm{F}^{-}$stubble. $\square=\mathrm{F}^{-}$sprout.

metodologia requer um conhecimento prévio das taxas de crescimento da espécie, homogeneidade genética, sintomatologia e sensibilidade ao poluente avaliada por respostas anátomo-morfológicas, fisiológicas ou bioquímicas específicas, facilmente mensuráveis. No caso específico de $\mathrm{F}^{-}$, um dos métodos protocolados e amplamente aceito recomenda o uso de L. multiflorum ssp. italicum var. lema cultivado em pequeno volume de substrato comercial e com capacidade de responder ao poluente em curto prazo. Foram testadas diversas espécies de gramíneas com rápido crescimento e ampla distribuição em regiões tropicais com o intuito de serem introduzidas como bioindicadores em regiões impactadas por emissões de flúor. Para otimizar o crescimento das plantas, no lugar de um substrato comercial (VDIRichtlinie 3792, 1982), foi empregado um Lato-solo Vermelho, freqüente na região do Cerrado, corrigido e adubado de acordo com as especificações de cultivo para gramíneas forrageiras. Os resultados apresentados permitem ampliar as opções de espécies bioindicadoras da presença de flúor em regiões tropicais e o potencial 


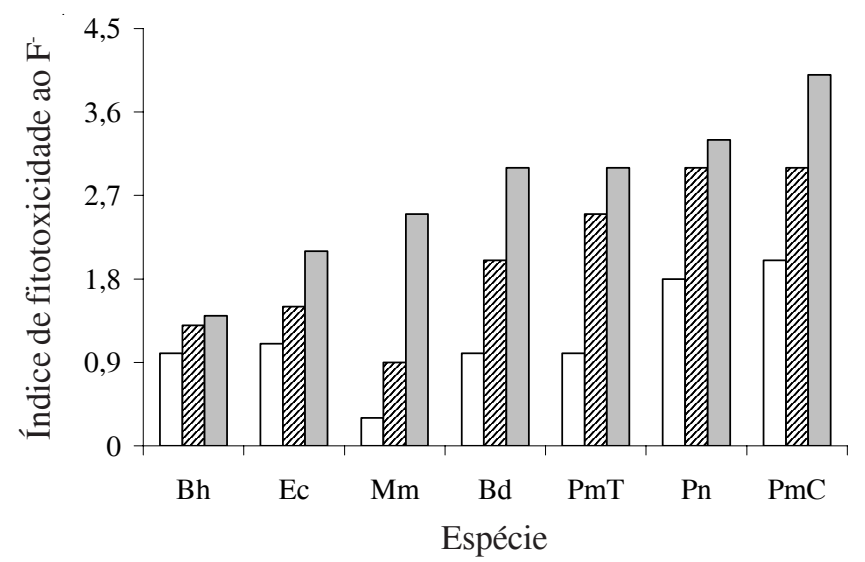

Figura 3. Evolução das injúrias foliares em gramíneas

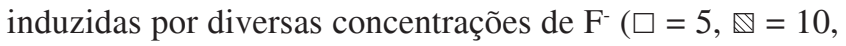
= $=15 \mathrm{~g} \cdot \mathrm{m}^{-3}$ ) aplicadas mediante chuvas simuladas durante 20 dias. $\mathrm{Bh}=B$. humidicola, $\mathrm{Pn}=P$. notatum, $\mathrm{Bd}=B$. decumbens, $\mathrm{Mm}=M$. minutiflora, $\mathrm{PmC}=P$. maximum cv. Colonião, PmT = P. maximum cv. Tobiatã, Ec = E. curvula . As espécies omitidas não apresentaram injúria.

Figure 3. Grasses foliar damages progress induced by different $\mathrm{F}^{-}$concentration $\left(\square=5, \mathbb{\nabla}=10, \square=15 \mathrm{~g} \cdot \mathrm{m}^{-3}\right)$ applied as simulated rain during 20 days. $\mathrm{Bh}=B$. humidicola, $\mathrm{Pn}=P$. notatum, $\mathrm{Bd}=B$. decumbens, $\mathrm{Mm}=M$. minutiflora, $\mathrm{PmC}=P$. maximum cv. Colonião, $\mathrm{PmT}=P$. maximum $\mathrm{cv}$. Tobiatã, Ec $=$ E. curvula . The omitted species did not show signs of injures.

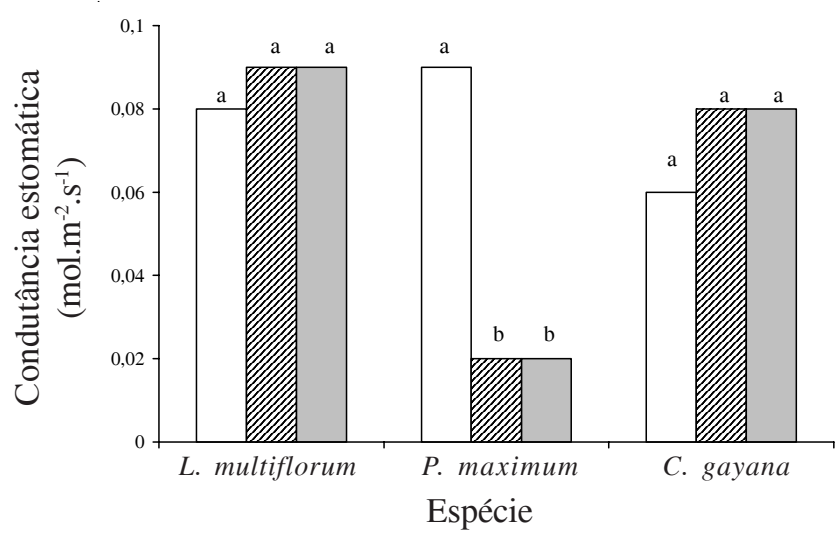

Figura 4. Efeito de diversas concentrações de $\mathrm{F}^{-} \quad(\square=0$, $\mathbb{\nabla}=10, \square=15 \mathrm{~g} \cdot \mathrm{m}^{-3}$ ) aplicadas mediante chuvas simuladas durante 20 dias sobre a condutância estomática $\left(\mathrm{g}_{\mathrm{s}}\right)$ de L. multiflorum ssp. italicum, P. maximum cv. Colonião e C. gayana. Colunas seguidas da mesma letra não diferem estatisticamente a $\mathrm{p}<0,05$ (teste de Tukey) dentro das espécies.

Figure 4. Effect of different F- concentrations $(\square=0, \mathbb{\nabla}=10$, - $=15 \mathrm{~g} \cdot \mathrm{m}^{-3}$ ) applied as simulated rain, during 20 days, on stomatal conductance $\left(\mathrm{g}_{\mathrm{s}}\right)$ of L. multiflorum ssp. italicum, $P$. maximum cv. Colonião and $C$. gayana. Bars followed by same letters does not differ statiscally at $\mathrm{p}<0,05$ (Tukey's test) within the species.

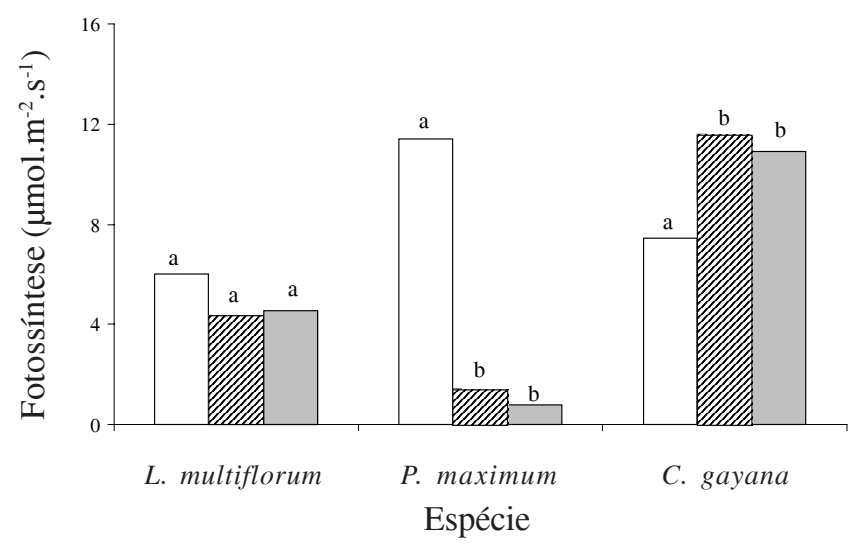

Figura 5. Efeito de diversas concentrações de $\mathrm{F}^{-} \quad(\square=0$, $\mathbb{Q}=10, \square=15 \mathrm{~g} \cdot \mathrm{m}^{-3}$ ) aplicadas mediante chuvas simuladas durante 20 dias sobre a fotossíntese (A) de L. multiflorum ssp. italicum, P. maximum cv. Colonião e C. gayana. Colunas seguidas da mesma letra não diferem estatisticamente a $\mathrm{p}<0,05$ (teste de Tukey) dentro das espécies.

Figure 5. Effect of different $\mathrm{F}^{-}$concentrations $(\square=0, \mathbb{\mathbb { N }}=10$, $=5 \mathrm{~g} \cdot \mathrm{m}^{-3}$ ) applied as simulated rain, during 20 days, on photosynthesis (A) of L. multiflorum ssp. italicum, $P$. maximum cv. Colonião and $C$. gayana. Bars followed by same letters does not differ statiscally at $\mathrm{p}<0,05$ (Tukey's test) within the species.

dessas espécies para serem empregadas como biosensoras, bioacumuladoras ou biointegradoras (Temmerman et al. 2004). Quando comparada com as outras nove espécies, L. multiflorum ssp. italicum mostrou baixas taxas de crescimento e pouca capacidade de rebrota sob condições de alta temperatura, incidência de radiação e umidade relativa, o que poderia significar a falta de adaptação dessa espécie às condições tropicais (tabela 2). Quando todas as espécies e variedades foram expostas ao fluoreto, a maioria delas acumulou $\mathrm{F}^{-}$no material vegetal em níveis muito superiores a $30 \mathrm{mg} \cdot \mathrm{g}^{-1} \mathrm{MS}$, considerado como padrão de referência para proteção de espécies sensíveis ao fluoreto em culturas padronizadas de L. multiflorum ssp. italicum (VDI-Richtlinie 2310, 1989). Apenas uma espécie, B. humidicola, não absorveu $\mathrm{F}^{-}$em níveis considerados tóxicos. Vários estudos confirmam que não somente espécies, mas variedades, cultivares e indivíduos de uma mesma espécie apresentam diferenças marcantes quanto ao grau de susceptibilidade a $\mathrm{F}^{-}$, entretanto, não era esperado que gramíneas de regiões tropicais apresentassem tal capacidade de acúmulo.

Plantas de C. gayana apresentaram a mesma tolerância de L. multiflorum ssp. italicum, porém, com um crescimento da rebrota maior, o que favorece seu uso como planta indicadora por períodos maiores. 


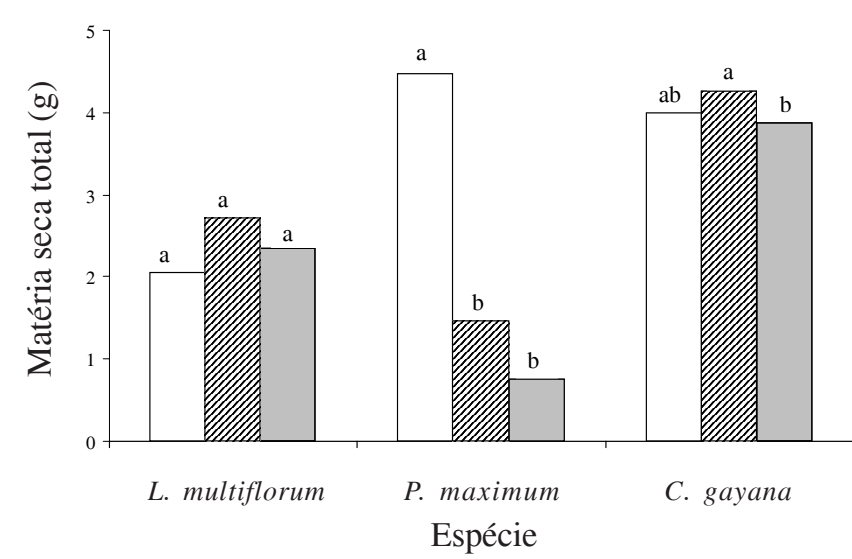

Figura 6. Efeito de diversas concentrações de $\mathrm{F}^{-} \quad(\square=0$,

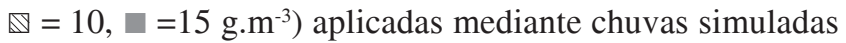
durante 20 dias sobre a produção de matéria seca total de L. multiflorum ssp. italicum, P. maximum cv. Colonião e C. gayana. Colunas seguidas da mesma letra não diferem estatisticamente a $\mathrm{p}<0,05$ (teste de Tukey) dentro das espécies.

Figure 6. Effect of different $\mathrm{F}^{-}$concentrations $(\square=0, \mathbb{\mathbb { N }}=10$, - $=15 \mathrm{~g} \cdot \mathrm{m}^{-3}$ ) applied as simulated rain, during 20 days, on total dry matter yield of L. multiflorum ssp. italicum, P. maximum cv. Colonião and C. gayana. Bars followed by same letters does not differ statiscally at $\mathrm{p}<0,05$ (Tukey's test) within the species.

P. maximum cv. Colonião é uma planta típica de reação, altamente sensível e com excelentes sintomas para avaliação. Com ambas as espécies poderão ser estabelecidas relações razoavelmente seguras entre os níveis de contaminação com flúor no ambiente e a concentração do íon acumulado nas plantas.

$\mathrm{OF}^{-}$, fornecido às plantas mediante chuva simulada, atinge os espaços intercelulares através da cutícula e pode ser absorvido pelas células do mesofilo alcançando o simplasto, ou ser arrastado pela corrente transpiratória, via apoplasto, até as extremidades do xilema, onde é acumulado. Existem evidências de translocação de $\mathrm{F}^{-}$ tanto do caule quanto das raízes para as folhas, possivelmente, através de movimento passivo e com a corrente transpiratória (Treshow \& Anderson 1991). O teor de $\mathrm{F}^{-}$no sistema radicular de todas as espécies foi inexpressivo em relação à parte aérea, variando na faixa de 1 a $4 \mu \mathrm{g} . \mathrm{g}^{-1}$ (figura 2) sendo que, segundo Braen \& Weinstein (1985), o solo normalmente não é uma fonte de níveis tóxicos de $\mathrm{F}^{-}$para a maioria das plantas e os teores de $\mathrm{F}^{-}$no sistema radicular ao redor de 2 a $20 \mu \mathrm{g} \cdot \mathrm{g}^{-1}$ são considerados comuns. A possibilidade de absorção de $\mathrm{F}^{-}$pelas raízes parece menor em virtude da baixa retenção de $\mathrm{F}^{-}$pelo substrato utilizado (de 0,18 a $\left.0,69 \mu \mathrm{g} \cdot \mathrm{cm}^{-3}\right)$.
O padrão de distribuição de fluoreto nas plantas da maioria das gramíneas sugere que ele se acumula em tecidos com maior atividade metabólica, ou seja, na rebrota. A penetração do ânion no apoplasto de tecidos jovens é facilitada pela menor quantidade de cutícula, cera epicuticular e pela presença relativamente grande de pectinas (Kannan 1986). O crescimento vegetativo vigoroso e as altas taxas de movimento de água favorecem o maior acúmulo de $\mathrm{F}^{-}$nos ápices e nas margens das folhas terminais (Leece et al. 1982). A relação entre a dose de $\mathrm{F}^{-}$aplicada à planta e seu acúmulo nos tecidos jovens da rebrota foi descrito por Brandt (1981).

A ocorrência de danos visuais, independente da espécie, aconteceu quase na sua totalidade nos tecidos da rebrota. Os efeitos sobre folhas em desenvolvimento devem-se provavelmente à ação do $\mathrm{F}^{-}$nos estágios iniciais da síntese de porfirina e, por conseguinte, sobre a biossíntese de clorofila (VDI-Richtlinie 2310, 1989). As gramíneas mais sensíveis como $P$. notatum, P. maximum cv. Colonião e $P$. maximum cv Tobiatã apresentaram clorose e necrose mesmo com teores tão baixos quanto 4,7, 13,8 e 27,0 $\mu \mathrm{g} \cdot \mathrm{g}^{-1}$ de F- Diversos estudos sugerem que plantas sensíveis podem ser injuriadas quando a concentração foliar excede a $30 \mu \mathrm{g} . \mathrm{g}^{-1}$ de $\mathrm{F}^{-}$(Treshow \& Anderson 1991). Logo, as espécies $P$. maximum cv. Colonião e $P$. notatum podem ser consideradas exceções. Verificou-se, ainda, que as espécies $P$. maximum cv. Colonião, $P$. maximum. cv. Tobiatã e $E$. curvula, todas sensíveis, acumularam mais $\mathrm{F}^{-}$na parte aérea que as gramíneas mais resistentes.

A injúria foliar é um critério amplamente usado para a caracterização de condições de intoxicação de plantas (Arndt et al. 1990). Espécies extremamente sensíveis como Gladiolus gandavensis L. cv. snow princess e Tulipa gesneriana L. cv. blue parrot são freqüentemente empregadas como plantas indicadoras em programas de monitoramento ativo de fluoreto atmosférico. No Brasil as espécies tropicais Cordyline terminalis e Discorea sp. (CETESB 1993a, b), Hemerocallis sp. (Klumpp et al. 1995), gramíneas tropicais (Oliva et al. 1997), Gallesia gorazema, Genipa americana, Joannesia princeps, Peltophorum dubium e Spondias dulcis (Silva et al. 2000) vêm sendo avaliadas e utilizadas com o mesmo propósito. É provável que a injúria foliar se encontre associada à alteração da matriz lipídica e sua relação com as proteínas solúveis da membrana, influenciando suas funções metabólicas, alterando a atividade de enzimas associadas, tais como as $\mathrm{H}^{+}$-ATPases (Giannini et al. 1987, Façanha \& De Meis 1995). A inibição da atividade da $\mathrm{H}^{+}$-ATPase 
conduz a alterações do transporte secundário de vários nutrientes e na perda de metabólitos através da membrana plasmática.

Das 10 espécies de gramíneas testadas, apenas Panicum maximum cv. Colonião e Chloris gayana mostraram-se promissoras como plantas bioindicadoras e eventualmente biosensoras. Elas mostraram altas taxas de crescimento homogêneo em pequeno volume de substrato ( $\mathrm{CV}=11 \%$ e $18 \%$, respectivamente), homogeneidade genética, sensibilidade diferenciada ao flúor com efeitos (clorose e necrose) inequívocos e facilmente mensuráveis. As duas espécies mais promissoras, $P$. maximum e $C$. gayana, foram submetidas a diversas concentrações de $\mathrm{F}^{-}$e seus efeitos, novamente confrontados com L. multiflorum ssp. italicum, medidos com metodologia não invasiva como porometria para assimilação de $\mathrm{CO}_{2} \mathrm{e}$ fluorescência da clorofila $a$.

Analisados os fatores que afetam a produção de matéria seca nas três espécies (figuras 4, 5, 6), ficou demonstrado que houve um efeito significativo das concentrações crescentes de $\mathrm{F}^{-}$sobre a fotossíntese e produção de matéria seca apenas de $P$. maximum $\mathrm{cv}$ Colonião. Nesta espécie, os resultados mostraram uma clara relação entre o fechamento dos estômatos e a depressão na assimilação do $\mathrm{CO}_{2}$, que, por sua vez, inibe a formação de matéria seca. Ballantyne \& Glover (1981) relataram que o efeito acumulativo de flúor no material vegetal termina por inibir o crescimento da planta, principalmente, por um efeito sobre a fotossíntese e a produção de matéria seca. Entretanto, os resultados abriram possibilidades a outros questionamentos sobre os mecanismos que poderiam afetar a assimilação de $\mathrm{CO}_{2}$ e em última instância a rebrota. Medidas in vivo da fluorescência da clorofila-a permitem avaliar a injúria causada pelo $\mathrm{F}^{-}$nos fotossistemas e na cadeia de transporte de elétrons. Resultados não apresentados indicam que nos primeiros estádios da injúria é possível observar drásticas alterações na dissipação fotoquímica e não fotoquímica da energia na fase lenta da fluorescência, o que parece demonstrar que o fluoreto atua como desacoplador, acelerando o fluxo de elétrons pelo PS II (Boese et al. 1995). Depois das injúrias visíveis causadas pelo flúor, a diminuição do crescimento da rebrota é um dos parâmetros mais sensível que pode ser determinado em curto prazo de exposição das plantas ao poluente. Todas as espécies foram submetidas ao $\mathrm{F}^{-}$ após dois cortes das brotações, o que significa que uma terceira rebrota dependeria muito mais das taxas fotossintéticas do que da mobilização das reservas. Isto poderia explicar a melhor resposta ao $\mathrm{F}^{-}$de $P$. maximum cv. Colonião em comparação com L. multiflorum ssp. italicum, uma vez que a fotossíntese de $P$. maximum cv. Colonião foi a mais afetada.

Agradecimentos - Os autores agradecem o apoio financeiro prestado pelo Conselho Nacional de Desenvolvimento Científico e Tecnológico (CNPq, Processo N 522183/94 ), pela Fundação de Amparo à Pesquisa do Estado de Minas Gerais e pela Coordenação de Aperfeiçoamento de Pessoal de Nível Superior (Capes, Processo $N^{\circ}$ 64.93.0061.00).

\section{Referências bibliográficas}

ALVES, P.L.C.A. \& OLIVA, M.A. 1993. Reações da soja à chuva ácida e solo contaminado. Ambiente 7:34-39.

ALVES, P.L.C.A., OLIVA, M.A., CAMBRAIA, J., SANT'ANNA, R. 1990. Efeito da chuva ácida simulada e de um solo de Cubatão-SP sobre parâmetros relacionados com a fotossíntese e a transpiração de plantas de soja. Revista Brasileira de Fisiologia Vegetal 2:7-14.

ARNDT, U. \& SCHWEIZER, B. 1991. The use of bioindicators for environmental monitoring in tropical and subtropical countries. In Biological Monitoring. Signal from the environment (H. Ellemberg, ed.). Gate/GTZ, Braunschweig, p.199-260.

ARNDT, U., BILLEN, N., SEUFERT, G., LUDWIG, W., BORKHART, K. \& OHNESORGE, B. 1990. The Hohenheim long-term experiment visible injury responses. Environmental Pollution 68:355-366.

ARNDT, U., FLORES, F. \& WEINSTEIN, L.H. 1995. Fluoride effects on plants. Diagnosis of injury in the vegetation of Brazil. Universidade Federal do Rio Grande do Sul, Porto Alegre

BALLANTYNE, D.J. \& GLOVER, B.L. 1981. Chloroplast electron transport, protein and RNA in fluoride-treated pea shoots. Environment and Experimental Botany 21:83-88.

BOESE, S.R., MACLEAN, D.C. \& EL-MOGAZI, D. 1995. Effects of fluoride on chlorophyll $a$ fluorescence in spinach. Environmental Pollution 89:203-208.

BRAEN, S.N. \& WEINSTEIN, L.H. 1985. Uptake of fluoride and aluminum by plants grown in contaminated soils. Water, Air and Soil Pollution 24:215-223.

BRANDT, C.J. 1981 Untersuchungen über Wirkungen von Fluorwasserstoff auf Lolium multiflorum und andere Nutzpflanzen. LIS-Berichte 14:1-140.

BUSTAMANTE, M., OLIVA, M.A., SANT'ANNA, R. \& LOPES, N.F. 1993. Sensibilidade da soja ao flúor. Revista Brasileira de Fisiologia Vegetal 5:151-157.

CETESB. 1993a. Danos à vegetação por fluoretos gasosos em usinas de alumínio. Informe Técnico $\mathrm{N}^{\circ}$ 001/93. Companhia de Tecnologia e Saneamento Ambiental, São Paulo

CETESB. 1993b. Diagnóstico de danos à vegetação por fluoretos gasosos em Cajati-SP. Informe Técnico 003/93. Companhia de Tecnologia e Saneamento Ambiental, São Paulo. 
DOMINGOS, M., KLUMPP, A. \& KLUMPP, G. 1998. Air pollution impact on the Atlantic forest in the Cubatão region, SP, Brazil. Ciência e Cultura 50:230-236.

DOMINGOS, M., ALVES, E.S., BULBOVAS, P., CUNHA, A.L., LONGUI, E.L., LOURENÇON, D.A.C.B., MORAES, R.M., RINALDI, M.C.S. \& MAZZONI-VIVEIROS, S.C. 2004. Reaction of Caesalpinia echinata Lam., a Brazilian tree species, to urban air pollution stress. In Urban air pollution, bioindication and environmental awareness (A. Klumpp, W. Ansel \& G. Klumpp, eds.). Cuvillier Verlag, Göttingen, p. 279-287.

ELLENBERG, H. 1991. Bioindicator and biological monitoring. In Biological monitoring. Signal from the environment. Gate/GTZ, Braunschweig, p.13-74

FAÇANHA, A.R. \& DE MEIS, L. 1995. Inhibition of maize root $\mathrm{H}^{+}$-ATPase by fluoride and fluoroaluminate complexes. Plant Physiology 108:241-246,

GARCIA-CIUDAD, A., GARCIA-CAIADO, B. \& PONTONSAN EMETERIO, C. 1985. Determination of fluoride in plant samples by a potentiometric method and nearinfrared reflectance spectroscopy. Communications in Soil Science and Plant Analyze 16:1107-1122.

GIANNINI, J.L., PUSHNIK, J.C., BRISKIN, D.P. \& MILLER, G.W. 1987. Fluoride effects on the plasma membrane ATPase of sugar beet. Plant Science 53:39-44.

KANNAN, S. 1986. Physiology of foliar uptake of inorganic nutrients. Proceeding of the Indian Academy Science 96:457-70.

KLUMPP, A., KLUMPP, G. \& DOMINGOS, M. 1994. Plants as bioindicators of air pollution at the Serra do Mar near the industrial complex of Cubatão, Brazil. Environmental Pollution 85:109-116.

KLUMPP, G., KLUMPP, A., DOMINGOS, M. \& GUDERIAN, R. 1995. Hemerocallis as bioindicator of fluoride pollution in tropical countries. Environmental Monitoring and Assessment 35:27-42.

KLUMPP, A., DOMINGOS, M. \& KLUMPP, G. 1996a. Assessment of the vegetation risk by fluoride emissions from fertiliser industries at Cubatão, Brazil. The Science of the Total Environmental 192:219-228.
KLUMPP, A., KLUMPP, G. \& DOMINGOS, M. 1996b. Bioindication of air pollution in the tropics. GefahrstoffeReinhaltung der Luft 56:27-31.

KLUMPP, A., KLUMPP, G., DOMINGOS, M. \& SILVA, M.D. 1996c. Fluoride impact on native tree species of the atlantic forest near Cubatão, Brazil. Water, Air, and Soil Pollution 87:57-71.

LEECE, D.R., SCHECTEMA, J.H. \& WEIR, R.G. 1982. The use of leaf analysis to diagnose fluoride toxicity in grapes. In Fluoride emissions (F. Murray, ed.). Academic Press, New York, p.101-24.

OLIVA, M.A. FIGUEIREDO, J.G. \& SOUZA, M.M. 1997. Bioindicação do flúor mediante gramíneas tropicais. In Indicadores ambientais (H.L. Matos \& N.B. Maia, coord.). DCA-PUC, Sorocaba, p.191-204.

RUTHSATZ, B. \& WEY, H. 1991. Concept for a biological monitoring study. In Biological monitoring. Signal from the environment. Gate/GTZ ed., Braunschweig, p. 75-128.

SILVA, L.C., AZEVEDO, A.A., SILVA, E.A. M. \& OLIVA, M.A. 2000. Flúor em chuva simulada: sintomatologia e efeitos sobre a estrutura foliar e o crescimento de plantas arbóreas. Revista Brasileira de Botânica 23:383-391.

TEMMERMAN, L., BELL, J.N.B., GARREC, J.P., KLUMPP, A., KRAUSE, G.H.M. \& TONNEIJCK, A.E.G. 2004. Biomonitoring of air pollutants with plants considerations for the future. In Urban air pollution, bioindication and environmental awareness (A. Klumpp, W. Ansel \& G. Klumpp, eds.). Cuvillier Verlag, Göttingen, p.337-373.

TRESHOW, M. \& ANDERSON, F. 1991 Plant stress and air pollution. John Willey \& Sons, New York.

VDI. Richtlinie 3792. 1982. Messen der Immissions-Wirkdosis von gas-und staubförmigem Fluorid in Pflanzen mit dem Verfahren der standardisierten Graskultur.. Blatt 2. VDIHandbuch Reinhaltung der Luft. Verein Deutscher Ingenieure, Düsseldorf, p.1-6.

VDI. Richtlinie 2310. 1989. Maximale Immissionswerte zum Schutze der Vegetation; Maximaler Immissionswerte für Fluorwasserstoff. In VDI-Handbuch Reinhaltung der Luft. Verein Deutscher Ingenieure, Düsseldorf, p.1-48.

WEINSTEIN, L.H. \& HANSEN, K.S. 1988. Relative susceptibilities of brazilian vegetation to airborne fluoride. Pesquisa Agropecuária Brasileira 23:1125-1137. 